\title{
Saúde, Desenvolvimento Econômico e Desigualdade nos Municípios do Rio Grande do Sul: um Estudo sobre Gasto Público eldese
}

\author{
Health, Economic Development and Inequality in the \\ Municipalities of Rio Grande do Sul: Study on Public Spending \\ and ldese
}

\author{
Janice Dornelles de Castro* \\ Maria Lecticia de Pelegrini** \\ Kalila Luize Balen Winkler"**
}

\begin{abstract}
Resumo: A saúde é um fator intrínseco ao desenvolvimento social e econômico, sendo ao mesmo tempo um determinante, uma medida e um resultado do progresso. Este estudo pretende analisar a capacidade das políticas públicas de financiamento do Sistema Único de Saúde (SUS) de contribuir para o desenvolvimento econômico e diminuir as desigualdades em saúde, observando a relação entre o gasto per capita com saúde e o Idese, no período de 2007 a 2010, nos municípios e nos Coredes do Rio Grande do Sul.O estudo conclui que o aumento do gasto per capita em saúde não reflete imediata melhora no Idese, portanto as desigualdades entre as regiões do estado se acentuam.
\end{abstract}

Palavras-chave: Economia da saúde. Desenvolvimento econômico. Equidade.

\begin{abstract}
Health is an intrinsic factor to social and economic development, being at the same time a determinant, a measure and a result of progress. The study aims to analyze the health system funding and its contribution to economic development and inequalities reduction in health, analyzing the relationship between spending on health care and the Idese, from 2007 to 2010, in the municipalities and Coredes of Rio Grande do Sul. The study concludes that the increase in the per capita health spending doesn't reflect immediate improvement in Idese, the inequalities between the regions of the State vowels.
\end{abstract}

Keywords: Health economy. Economic development. Equity.

JEL Classification: I18; I10; 01.

\footnotetext{
- Professora Doutora da Faculdade de Ciencias Econômicas da Universidade Federal do Rio

- Trande do Sul (UFRGS).E-mail: janice.castro@ufrgs.br

*.* Grénica da Secretaria Estadual de Saúde do Rio Grande do Sul. E-mail: mimpelegrini@terra.com.br E-mail; kalila_luize@hotmail.com
} 


\section{Introdução}

O desenvolvimento econômico dos países foi medido durante longo período através do indicador do nível de renda per capita, sendo empregado tanto para medir a riqueza, quanto para medir o bem-estar social. Atualmente propõe-se que o conceito de desenvolvimento seja "[...] entendido não apenas como crescimento da produção, mas também abrangendo mudanças estruturais necessárias para torná-lo sustentável a longo prazo, e mais justo do ponto de vista distributivo" (MOLLO, 2013).Deve tratar, portanto, das questōes de justiça social, distribuição da renda, acesso à educação, saúde, água, saneamento, emprego, terra e consumo, bem como do empoderamento dos cidadãos para influenciar os rumos da sociedade. De acordo com a United Nations Research Institute for Social Development (2013), a saúde é um fator intrínseco ao desenvolvimento social e econômico, sendo ao mesmo tempo um determinante, uma medida e um resultado do progresso. O principal desafio dos sistemas de saúde, nesse caso, é garantir a distribuição equitativa de cuidados e, assim, a saúde das pessoas. No entanto, essas condições são muitas vezes produzidas fora dos sistemas de saúde, sendo resultado do conjunto das políticas públicas, como educação, agricultura, transporte, meio ambiente e outras políticas econômicas e sociais (UNITED NATIONSRESEARCHINSTITUTE FOR SOCIAL DEVELOPMENT, 2013), reduzindo, assim, a capacidade das políticas públicas de saúde influenciarem exclusivamente as condições de bem-estar e saúde.

Na mesma direção, Sen (2002) aponta o pensamento de saúde como de liberdade.Para o autor, é possível pensar que o desenvolvimento deve considerar a garantia das necessidades sociais básicas vinculadas à garantia de bem-estar social, articulando o crescimento econômico sustentável com equidade e justiça social. Considerando essas perspectivas, parece claro a indicação da necessidade de políticas públicas convergentes, cujos objetivos sejam a criação de condições econômicas e sociais que promovam o bem-estar.

\section{Desenvolvimento Econômico e Saúde}

A discussão sobre a implicação do campo da saúde para o desenvolvimento econômico é apresentada na literatura nacional por Duarte de Araújo (1975).O autor apresenta uma revisão da produção teórica sobre o tema na literatura nacional e internacional nas décadas de 1950, 1960 e metade da década de 1970. A discussāo sobre o entendimento da contribuição da saúde como determinante ou determinada pelo desenvolvimento econômico é apresentada de forma inconclusiva. 
O autor apresenta contribuiçōes feitas por Myrdall (1952 19 apud DUARTE DE ARAÚJO, 1975, p. 516), que discute o círculo vicioso da pobreza: causalidade circular cumulativa (dinâmica inter-relacional) de saúde e crescimento econômico. O autor afirma que os investimentos em saúde fazem parte do desenvolvimento econômico e não podem ser analisados isoladamente. A contribuição é dada, ainda, no sentido da necessidade de serem feitas análises globais, assumindo a defesa de que a saúde é pré-condição ao desenvolvimento econômico.

O artigo é rico por apresentar as discussōes feitas durante a conferência sobre serviços em saúde de 1962, quando foram apresentadas as primeiras formulaçōes sobre capital humano. Autores como Fein $\left(1962^{2}\right.$ apud DUARTEDE ARAÚJO,1975, p. 516) apresentam para discussão a questão do valor econômico da vida humana, criticando enfaticamente a abordagem malthusiana e reforçando a análise do desenvolvimento econômico para além do crescimento de renda per capita como melhoria da qualidade de vida.

O argumento em relação à melhoria da qualidade de vida relaciona-se com a queda da mortalidade, mas também com a diminuição da morbidade, visto que essa redução pode ter como resultado o aumento do produto nacional, através do crescimento da produtividade da economia, seja pela diminuiçăo do absenteísmo, aumentando o número de homens/horas trabalhadas, seja pelo aumento da eficiência da força de trabalho. Outro aspecto dessa reflexão discute a capacidade de a saúde influenciar a melhoria das condiçōes de aprendizagem e, com isso, afetar o desenvolvimento econômico. $O$ artigo apresenta, ainda, contribuições de Mushkin (1962 33 apud DUARTEDE ARAÚJO, 1975, p. 518), Bravo et al. (1963 apud DUARTEDE ARAÚJO,1975, p. 518) e Horwitz (1962 ${ }^{5}$ apud DUARTE DE ARAÚJO,1975, p. 518), no sentido de tratar a saúde como investimento, sugerindo incluir essa área nos planos de desenvolvimento dos países, alertando para a necessidade de se melhorar os registros e sistemas de informação para a avaliação de programas de saúde.

Drewnowski (1966' apud DUARTE DE ARAÚJO, 1975, p. 518), em sua análise de desenvolvimento econômico, propōe a criação de indicadores de fluxo e de estoque. Ele considera a saúde como parte do capital social da sociedade - espe-

I MYRDAL, G. Les aspects économiques de la santé. Chron. Org. mond. Santé, v. 6, p.224-242, 1952.

2 FEIN, R. Health programs and economic development. In: AXELROD, S. J. (Ed.). The economics of health and medical care: proceedings of the first Conference on the Economics of Health Services. Ann Arbor: The University of Michigan, 1964. p. 217-282.

3 MUSHKIN, S. J. Health as an investment. Journal Political Economy, v. 70, n. 5, Pt. II, p. 129-157, 1962.

4 BRAVO, A. L. et al. Desenvolvimento sరcio-econơmico e planificação em saúde. Revista Brasileira Malar, v. 15, p. 299-309, 1963.

5 HORWITZ, A. El proceso de programar en salud y sus relaciones con el desarrollo económico. Boletín de la Oficina Sanitaria Panamericana, v. 55, n. 5, p. 472-479, nov. 1963.

6 DREWNOWSKI, J. Les facteurs économiques et sociaux du développement. Genève: Institut de Recherche des Nations Unies pour le Développement Social, 1966. (Rapport $n^{\circ} .3$ ). 
cialmente quando analisada sob a perspectiva dos serviços à disposição da população -, bem de consumo dos indivíduos e medida do nível de renda e medida do nível de bem-estar. $O$ autor propōe também indicadores para avaliação do nível de vida diretamente relacionados com a intervenção de saúde como acesso aos serviços de saúde, óbitos por doenças infecciosas e taxa de mortalidade da população acima de 50 anos.

Em 1967, em Punta del Este, Uruguai, elaborou-se a Declaração dos Presidentes de Países Americanos (19677 apud DUARTE DE ARAÚJO,1975, p. 519), que conclama a necessidade de melhoria das condiçôes de vida para o desenvolvimento econômico. Em 1968, o Instituto de Pesquisas para o Desenvolvimento Social das Nações Unidas (1968 apud DUARTE DE ARAÚJO, 1975, p. 519) chamou atenção para a correlação entre esperança de vida ao nascer e indicadores econômicos. Nesse período, a discussăo sobre a insuficiência dos indicadores de assistência médica e as desigualdades de acesso, como fator isolado para medir a qualidade de vida, já estavam colocadas. Essas condiçôes trazem sempre a discussão da capacidade da assistência médica, como fator isolado da ação de saúde, resultar na melhoria da qualidade de vida da população.

Evoluindo nessa discussão, em 1970 Granahan et al. (1970 apud DUARTEDE ARAÚJO, 1975, p. 519) propuseram o Índice Sintético de Desenvolvimento. Nessa proposta foram elencados 72 indicadores sociais e econômicos que apresentaram uma forte correlação em 18 deles, tais como esperança de vida ao nascer e consumo diário de proteína animal. Em 1971, Griffith et al. (1971 ${ }^{10}$ apud DUARTEDE ARAÚJO, 1975, p. 520) apresentaram um trabalho revisitando a discussão sobre a contribuição da saúde para o desenvolvimento económico, identificando os efeitos negativos da doença sobre o crescimento econômico e como a melhoria do nível de saúde pode interferir nos níveis de desenvolvimento econômico.

A ausência de consenso nessa discussão pode ser percebida na posição de Scott (1971 11 apud DUARTE DE ARAÚJO, 1975, p. 520), que, em 1971, criticou alguns estudos sobre o impacto dos níveis de saúde sobre o crescimento econômico e afirmou que não levavam em conta a influência dos níveis de saúde no crescimento econômico e vice-versa, chegando à conclusão de que os indicadores sociais poderiam variar independente do crescimento econômico. Em contrapon-

7 ORGANIZATION OF AMERICAN STATES. Declaration of the Presidents of American. Punta del Este, 1967.

8 UNTTED NATIONS. Research Institute for Social Development. Geneva, 1968. (Research Notes, n. 1)

9 GRANAHAN, D. V. et al. Contens and measurement of socio-economic development: an empirical enquiry. Geneva: United Nations Research Institute for Social Development, 1970.

10 GRIFFITH, D. H.; RAMANA, D. V.; MASHAAL, H. Contribution of health to development. International Journal Health of Services, v. 1, n. 3, p. 253-279, 1971.

II SCOTT, W. Cross national studies of the impact of levels of living on economic growth: an example. International Joumal Health of Services, v. 1, n. 3, p. 225-232, 1971. 
to a essa posiçăo, no mesmo ano, Navarro (1971 ${ }^{12}$ apud DUARTE DE ARAÚJO, 1975, p. 522) publicou, no International Journal of Health Services, um trabalho demonstrando a incapacidade de os modelos clássicos medirem o desenvolvimento econômico, já que não incluem indicadores sociais como fatores explícitos nas funções de produção, evidenciando mais uma vez os aspectos políticos implicados nessa percepção.

No Brasil, a discussão sobre a contribuição da saúde para o desenvolvimento pode ser analisada no relatório da Comissão Nacional de Bem-Estar Social $\left(1954^{13}\right.$ apud DUARTE DE ARAÚJO, 1975, p. 523). Nesse estudo, a saúde é colocada como uma variável dependente do desenvolvimento econômico, e é feita uma crítica à tendência de construir uma "superestrutura médica que o país não suporta". A ação das políticas públicas, através do Ministério da Saúde, reforça os riscos de dispersão de recursos, múltiplas açōes e falta de organização na formação de profissionais, reforçando as açōes de saúde como consumo.

Essa dualidade sobre a contribuição da saúde ao desenvolvimento econômico persiste nas abordagens das políticas do setor. Mello (1963 ${ }^{14}$ apud DUARTE DE ARAÚJO, 1975, p. 523), em defesa da visão de saúde como consumo, afirma que "[...] assistência médica é função da renda [...]" e que investir recursos em saúde pode atrapalhar o desenvolvimento em saúde.

Contraposiçōes feitas por autores como Coutinho et al. (1963 ${ }^{15}$ apud DUARTE DE ARAÚJO, 1975, p. 523) mostram a correlação entre indicadores de saúde e de desenvolvimento, mas apontam o limite dos trabalhos pela ausência de estatísticas confiáveis. Por outro lado, autores como Ferreira (1963 ${ }^{16}$ apud DUARTE DE ARAÚJO, 1975, p. 524) defendem a saúde enquanto alicerce do desenvolvimento, assim como Novaes (1964 $1971^{18}$ apud DUARTE DE ARAÚJO, 1975, p. 524), que discute a saúde como variável dependente do desenvolvimento.

No pós-Segunda Guerra, os diferentes Estados de bem-estar social implantados nos países da Organização para a Cooperação e Desenvolvimento Económico (OCDE) contribuíram para a reduçăo da pobreza e melhoraram a saúde da populaçāo. No final dos anos 70, esses modelos se tornaram mais individualistas, fenô-

12 NAVARRO, V. Health and socio-economic development. International Journal Health of Services, v. 1 , n. 3, p. $187-188,1971$.

13 CASTRO, A. et al. Saúde pública e desenvolvimento econômico. Relatório da Subcomissáo de Saúde da Comissáo Nacional de Bem-Estar Social. Revista Hig. Saúde Pública, Rio de Janeiro, v. 13, p. 53-64, 1954 .

14 MELLO, C. G. Assistência médica e desenvolvimento econômico. Revista Brasileira Malar., v. 13, p. 291-297, 1963.

15 COUTINHO, J. O. et al. Problemas médico-sanitários de áreas subdesenvolvidas. Revista Brasileira Malar., v. 15, p. 157-189, 1963.

I6 FERREIRA, M. J. Perspectivas atuais da saúde pública. Revista Brasileira Malar., v. 15, p. 505-513, 1972.

17 NOVAES, M. Integración de los programas de salud en la política de desarrollo. Boletín de la Oficina Sanitaria Panamericana, v. 57, p. 117-138, 1964.

18 NOVAES, M. Saúde e desenvoluimento econômico. Salvador: Ediçăo CPE, 1961. 
meno que promoveu, em países de baixa e média renda, um problema de dívidas, empréstimos e financiamentos com países de alta renda e instituiçōes financeiras, criando muitas dificuldades para o estabelecimento de um sistema de saúde igualitário (BLAS, 2008). Durante um longo período,a discussão sobre o desenvolvimento económico e a saúde perde força, sendo subordinada ao discurso e às teses neoliberais hegemônicos que priorizavam a discussăo da reorganização macroeconômica dos países, do papel do Estado ou do Estado mínimo, a reorientaçáo do gasto público, a modernização da gestão e das políticas de privatizaçăo (FLEURY, 2009;GALLO, 2005; MACIEIRA, 2007; MENICCUCCI, 2007; SANTOS, 2008;).

Apenas nos anos 2000 retoma-se a discussão do desenvolvimento econômico e da saúde. Para Mollo (2013), "O interesse crescente pelo tema desenvolvimentismo, após três décadas de arquivamento acadêmico, tendo em vista a hegemonia ortodoxa neoliberal, é alvissareiro". Nesse período, muitas reformas nos sistemas de saúde foram propostas e eventualmente implementadas, visando à redução do papel do Estado.

De acordo com Jomo (2003), que apresenta um estudo sobre os efeitos da globalização e da liberalização da economia em cinco países asiáticos (Indonésia, Malásia, Coreia, Tailândia e Taiwan), o Consenso de Washington e as propostas do Banco Mundial criaram uma hegemonia do pensamento econômico em relação à eficiência das políticas de minimização do Estado para promover o crescimento econômico. As intervenções seletivas do Estado, em que pese tenham contribuído para um crescimento rápido, mudanças estruturais, desenvolvimento da capacidade de competição internacional da indústria e aumento do emprego e dos salários médios, não tiveram consequências diretas na diminuiçăo da desigualdade ou na melhoria da distribuiçăo de renda. O pequeno investimento na criaçăo de redes de proteção social, para se contrapor às rápidas mudanças estruturais e aos ciclos econômicos, promoveu um desastre social quando ocorreram a crise monetária e financeira de 1997 e a recessão que se seguiu, evidenciando inadequação dessa política.

Os objetivos do milênio para 2015 propostos pela ONU consideram como requisito para o desenvolvimento econômico e social o investimento em saúde, ciência e tecnologia. De acordo com Morel (2004), esse deve ser o grande desafio para os países em desenvolvimento, pois será necessário organizar sistemas de pesquisas em saúde baseados em prioridades sanitárias e, ainda, assegurar a incorporação dos resultados na política e nas ações de saúde. No Brasil, o forte parque industrial e a vigorosa comunidade científica deverá contribuir para se alcançar esses propósitos.

Para Gadelha (2006), todos os países que se desenvolveram e passaram a competir em melhores condiçōes com os mais avançados associaram uma indústria forte com uma base endógena de conhecimento, aprendizado e inovação. 
Utilizando dados de comércio exterior, o autor demonstra como a desconsideração da lógica do desenvolvimento nas políticas de saúde levou a uma situação de vulnerabilidade econômica do setor que pode limitar os objetivos de universalidade, equidade e integralidade. Nesse contexto, um país que pretende chegar a uma condição de desenvolvimento e de independência requer, ao mesmo tempo, indústrias fortes e inovadoras e um sistema de saúde inclusivo e universal.

Após um período de poucas discussóes, o atual momento é de muitas propostas em relação ao conceito de desenvolvimento econômico e saúde e sua relação com a desigualdade. A Comissão de Determinantes Sociais da Saúde da Organização Mundial da Saúde (WORLD HEALTH ORGANIZATION, 2008) propõe uma nova visão para o desenvolvimento econômico que sugere que saúde e equidade em saúde devem ser o principal resultado das políticas sociais e, em que pese a importância do crescimento econômico, especialmente para os países pobres, não é suficiente para garantir equidade nos resultados das políticas sociais. Assim, a comissāo propōe um modelo para explicar o que determina as desigualdades em saúde.

Em relação a essa discussão, Mirvis (2008) trata dos motivos pelos quais a melhora na saúde gera desenvolvimento econômico, focando a relação entre saúde da população e sua produtividade econômica. Para comprovar essa relação, sāo feitas análises de períodos da história econômica, ficando evidente que vários dos booms econômicos estão associados ao controle de doenças e à melhora na nutrição. Nesse caso, saúde é uma commodity macroeconômica, e a infraestrutura do sistema de saúde de países em desenvolvimento é um pré-requisito para estimular o desenvolvimento econômico, assim como a boa saúde da população é de vital importância para a reduçăo da pobreza e para o crescimento econômico de curto e longo prazo. Mirvis (2008) acredita que a produtividade econômica está diretamente relacionada à saúde por causa de quatro fatores principais:

a) doenças reduzem o bem-estar econômico dos indivíduos, pela reduzida renda familiar, gasto com cuidados de saúde, perdas de rendas futuras, redução de expectativa de vida e diminuição da produtividade, devido ao estado físico e psicológico do indivíduo;

b) falta de saúde leva a consequências no futuro, pois doenças durante a gestação, ou na primeira infância, geram efeitos nas habilidades cognitiva e psicológica, podendo limitar a educaçâo. Além disso, a doença de uma geração pode influenciar diretamente na geração futura, pois pais doentes năo oferecem apoio necessário para a educação;

c) altas taxas de mortalidade infantil e de fertilidade levam familias mais pobres a compensar a alta taxa de mortalidade, tendo um maior número de filhos, restando pouca renda para ser gasta por filho; 
d) falta de saúde em uma localidade gera consequências para toda a sociedade, pois reduz investimentos externos, turismo, afeta a força de trabalho e mobiliza os escassos recursos, deixando outras esferas sem cobertura.

Mirvis (2008) discute, ainda, que o valor econômico do aumento na expectativa de vida é tão grande quanto o valor de todos os outros bens de consumo e serviços juntos. Cada ano de vida perdido devido à morte prematura leva a uma perda econômica equivalente a três vezes a renda anual desse indivíduo, sendo que o aumento de $10 \%$ na expectativa de vida está associado a um crescimento econômico de 0,3 a $0,4 \%$ por ano. Pode-se concluir que existe uma forte relação entre saúde e desenvolvimento econômico, mas como garantir a diminuição das desigualdades relacionadas com a saúde e obter um desenvolvimento econômico que seja socialmente justo?

Com objetivo de esclarecer essa questăo, inúmeros autores discutem a diferença entre desigualdade em saúde e a desigualdade social em saúde. A desigualdade em saúde é a ausência de diferenças em saúde, injustas e inevitáveis entre grupos populacionais definidos social, demográfica e geograficamente. As desigualdades sociais em saúde, por sua vez, referem-se às distintas oportunidades e recursos relacionados com a saúde que as pessoas acessam em função de sua classe social, sexo ou etnia. Essas diferenças de oportunidades tendem a aumentar já que a saúde melhora mais rápido para os indivíduos de classes sociais mais elevadas.Entretanto, essa tendência poderá ser alterada se forem aplicadas as intervençöes e políticas públicas sanitárias e sociais adequadas (BORRREL; ARTAZCOZ, 2008; FLEURBAEY, 2009; MARMOT, 2007).

Segundo Fleurbaey (2009), num mesmo grupo social existem diferenças em saúde que podem ser completamente identificadas pelas diferenças nos hábitos dos indivíduos, como, por exemplo,ser tabagista.Essas desigualdades são legítimas. As diferenças no uso dos serviços de saúde que refletem na diferença de necessidades, por sua vez, são desejáveis. Existem desigualdades legítimas e nãolegítimas, sendo legítimas (justas) as que dependem de responsabilidade individual. Por isso, as diferentes visões sobre equidade são apenas uma questão do que a pessoa considera como legítimo ou ilegítimo.

Ainda em relaçăo à discussão da necessidade de construçăo de um modelo de desenvolvimento econômico socialmente justo, Borrell (2008) apresenta um modelo para avaliar desigualdades, proposto pela Comissáo de Determinantes Sociais da Saúde,como sendo composto por um conjunto de determinantes: a) estruturais, relativos ao contexto socioeconômico e à posiçăo socioeconômica; $\mathrm{e}$ b) intermediários, relativos às circunstâncias materiais, psicossociais e aos fatores comportamentais e biológicos (BORRREL; ARTAZCOZ, 2008). Blas (2008), por sua vez, apresenta outra proposta da Comissão de Determinantes Sociais da Saúde sobre os papéis que o Estado e a sociedade devem desempenhar para gerar maior 
equidade em saúde, segundo nove redes de conhecimento (nine knowledge networks). São elas: desenvolvimento da primeira infância, condiçōes de emprego, sistemas de saúde, contexto urbano, globalização, exclusāo social, igualdade de gêneros, condições de saúde pública prioritárias e mensuração e evidências. Com base nisso, os governos podem agir como provedores dos direitos humanos e de serviços essenciais, facilitadores de políticas que forneçam bases para o desenvolvimento da equidade em saúde e coletores e monitores de dados sobre sua populaçăo que forneçam informaçōes sobre equidade em saúde. A sociedade tem um papel fundamental, segundo Blas (2008), para a redução da desigualdade. O engajamento das comunidades nas decisōes é mais apropriado e efetivo, indicando o que a população prefere e precisa. Para que esses grupos da sociedade funcionem melhorando o sistema, é necessário que o governo reconheça a legitimidade política da sociedade civil e a voz da comunidade, designando políticas que thes deem real poder.

Para Fleurbaey (2009), a igualdade no uso dos recursos de cuidados em saúde não determina a igualdade nos cuidados de saúde, pois o que realmente importa são os impactos desses cuidados de saúde. Por isso, a preocupaçăo deve ser sobre a composição do consumo em saúde, mesmo que ouso dos recursos seja igualitário. Se uma pessoa adquire tratamento de qualidade inferior, devido a fatores que não são de sua responsabilidade, é difficil sustentar que não há injusta desigualdade em saúde. Porém, remover as desigualdades em cuidados de saúde não é suficiente para remover completamente as desigualdades em saúde.

No Brasil, a saúde como direito e como dever do Estado surgiu nos anos 1930 com o modelo nacional-desenvolvimentista. Nas décadas seguintes, a hegemonia das políticas de cunho liberal afetou a organizaçăo dos serviços de saúde. Atualmente, a nova escola desenvolvimentista propōe modelos econômicos dinâmicos, politicamente democráticos e socialmente inclusivos (VIANA, 2007). Os serviços de saúde no Brasil tiveram e ainda têm um papel relevante na organização da rede urbana e na delimitação territorial. A política de descentralização e regionalização do Sistema Único de Saúde (SUS) com os correspondentes investimentos federais insere a saúde no novo modelo de desenvolvimento brasileiro, historicamente marcado por desigualdades econômicas, sociais e territoriais (GADELHA, 2011).

Como visto anteriormente, a busca pela equidade é um processo cujo primeiro passo é o reconhecimento e detalhamento das desigualdades sociais (BLAS, 2008; BORRRE; ARTAZCOZ, 2008; FLEURBAEY, 2009; GADELHA, 2011; MIRVIS, 2008; VIANA, 2007). Portanto, é muito importante para o planejamento das políticas públicas a construção de um índice de condiçōes de vida e saúde (ICVD) que reflita a complexidade da realidade de saúde e doença. Luiz (2009) elabora esse índice, utilizando quatro dimensōes: econômica, consciência e conduta, ecológica e biológica. Para a elaboração do índice, utiliza como base seis indicadores: demo- 
grafia, renda, oferta e produçăo de serviços de saúde, meio ambiente e habitaçăo, educação (LUIZ, 2009).

A proposta do SUS é usar o planejamento para organizar e distribuir os serviços conforme a necessidade da população. Esses serviços são gratuitos e o atendimento é universal e organizado regionalmente. Os serviços são hierarquizados conforme a sua complexidade tecnológica. A rede básica é a porta de entrada do sistema e deve garantir o atendimento ambulatorial básico e o desenvolvimento das açōes de saúde pública. Nesse modelo, a saúde é considerada um direito do cidadão. O processo de descentralização da saúde, previsto quando da criação do SUS, tomou maior impulso a partir da promulgação da Constituição Federal em 1988 e da consequente aprovação das Leis $\mathrm{n}^{\circ} 8.080$ e $\mathrm{n}^{\circ} 8.142$, ambas de 1990 , as quais preveem, entre outras coisas, a descentralização e o repasse de recursos financeiros para os estados e os municípios (MÉDICI, 1995; PIOLA, 1995). A descentralização e o repasse foram realizados através da contínua absorçāo de novas e caras tecnologias que trouxeram a discussão sobre custos da atenção para a ordem do dia (CASTRO, 2000; SCHRAIBER, 1995; SILVA JÚNIOR, 1998). O impacto desse processo se refletiu em grandes investimentos em infraestrutura assistencial (obras e equipamentos) que tinham como característica ser marcadamente desigual entre as regiōes, favorecer a criação de serviços privados através de financiamento público e organizar um sistema de saúde mais focado na oferta de serviços que na demanda.

O Estado brasileiro tem sido um grande financiador das políticas de saúde desde a formação do país. A reforma do sistema proposta pela criação do SUS foi bem-sucedida em diversos aspectos, no entanto permanecem algumas questōes fundamentais a serem respondidas, como, por exemplo, como será possível garantir a equidade no acesso aos serviços de saúde com recursos financeiros limitados? Esses recursos limitados estão sendo usados para diminuir as desigualdades?

Sob a luz dessa discussão, este estudo pretende observar a capacidade das políticas públicas de financiamento do SUS de contribuir para o desenvolvimento econômico, social, soberano e sustentável e, assim, diminuir as desigualdades em saúde.

Para se atingir esse objetivo, propōe-se neste estudo a análise de apenas um aspecto: a relação entre o gasto per capita com saúde e o índice de desenvolvimento socioeconômico (Idese), bloco saúde, no período de 2007 a 2010, nos municípios e conselhos regionais de desenvolvimento (Coredes) do Rio Grande do Sul. Pretende-se verificar se os gastos realizados pelo SUS estão contribuindo para a reduçāo das desigualdades entre regiōes. 


\section{Materiais e Métodos}

O objetivo geral deste trabalho é verificar a capacidade da política de financiamento do SUS de diminuir as desigualdades em saúde nos municípios do Rio Grande do Sul. Os objetivos especíicos são: a) analisar a evolução da distribuição do gasto per capita em saúde no período de 2007 a 2010; b) verificar a relação entre a variação do gasto per capita com saúde e a variação do Idese saúde, no período entre 2007 e 2010; e c) analisar a desigualdade regional em relaçăo ao gasto per capita em saúde e o Idese saúde.

Os dados para a realização do estudo foram obtidos em diversas fontes: a) IBGE para os dados de população e indexador- neste caso foi utilizado o índice de preços ao consumidor ampliado (IPCA); b) Ministério da Saúde/Datasus- coletou-se informações do Sistema de Informações de Orçamento Público de Saúde (Siops) para se obter os dados de gasto per capita em saúde; c) Fundação de Economia e Estatística (FEE) - foram utilizados dados do Idese.

O tratamento dos dados foi realizado da seguinte forma: na primeira etapa, para a população, foram utilizados os dados referentes ao censo de 2000 , corrigidos para os anos subsequentes, usando-se a estimativa de crescimento do próprio IBGE. Os gastos per capita em saúde foram calculados a partir dos dados coletados no Siopse deflacionados pelo IPCA, ano base 2010. Foi calculada a taxa de variação dos gastos e do Idese saúde no período para cada município e Corede. Em relação ao Idese, as informações foram coletadas junto à FEE e calculou-se a taxa de crescimento no período de 2007 a 2010.

O Idese é um índice sintético que mede o grau de desenvolvimento dos municípios do estado do Rio Grande do Sul. Pode variar entre 0 (nenhum desenvolvimento) e 1 (desenvolvimento total). Abrange três blocos de indicadores sociais e econômicos, com a mesma ponderação: educação, saúde e renda. No estudo foi utilizado apenas o bloco da saúde, calculado a partir dos indicadores materno-infantil (mortalidade menor de cinco anos e número de consultas pré-natal),condições gerais de saúde (mortes por causas evitáveis e óbitos por causas mal definidas) e longevidade.Na segunda etapa do tratamento dos dados, utilizaram-se planilhas do software Excel para ordenar os municípios e os Coredes em relação ao gasto per capita e o Idese saúde para os anos 2007 a 2010 e calculou-se a variação dessas duas variáveis no período. Com base nessas informaçōes, foram elaborados gráficos e tabelas. 


\section{Resultados e Discussão}

Guimarães (2004) discute o uso de indicadores na avaliação de políticas públicas e a necessidade de cuidado nas análises e conclusões, pois existe o perigo de superdimensionamento da capacidade de mensuração da realidade, já que os conceitos são abstratos e possuem complexa inter-relação (desenvolvimento humano, condiçăo de vida, etc.). Além disso, a modificaçăo anual desses indicadores podem năo explicar ou implicar alteraçōes significativas da realidade que se propōem medir - seria necessário períodos longos de tempo para avaliar o impacto das políticas medidas por indicadores. Uma proposta metodológica de construção de indicadores de desigualdade foi testada por Drachler et al. (2003) e utilizada pela Pesquisa Avaliativa de Desigualdade em Saúde no Rio Grande do Sul, que avalia as desigualdades em saúde como diferenças na qualidade de vida e capacidades humanas socialmente determinadas. O estudo de Pelegrini (2005) aponta que as políticas redistributivas de recursos para a saúde utilizadas no Rio Grande do Sul tiveram a capacidade de beneficiar municípios de menor IDH-M no período estudado - 2001. Nesta pesquisa se propõe mensurar a eficácia das políticas de financiamento da saúde, através da relação entre o gasto per capita em saúde e o Idese bloco saúde.

Analisando a evolução da distribuição do gasto per capita em saúde no período de 2007 a 2010, pode-se observar um crescimento em praticamente todos os municípios do estado. No entanto, não parece haver relação com a variação do Idese saúde. O menor valor de gasto per capita em saúde foi de $\mathrm{R} \$ 85,98$, em 2007 , e de $R \$ 88,35$, em 2010 , enquanto que o maior valor foi de $R \$ 1.070,56$, em 2007 , e de $R \$ 1.580,94$, em 2010 , sendo que a média foi de $R \$ 360,56$, em 2007 , e de $R \$$ 447,40, em 2010. 
Gráfico 1 - Gasto per capita em saúde e variação do Idese saúde nos municípios do Rio Grande do Sul (2007-2010)

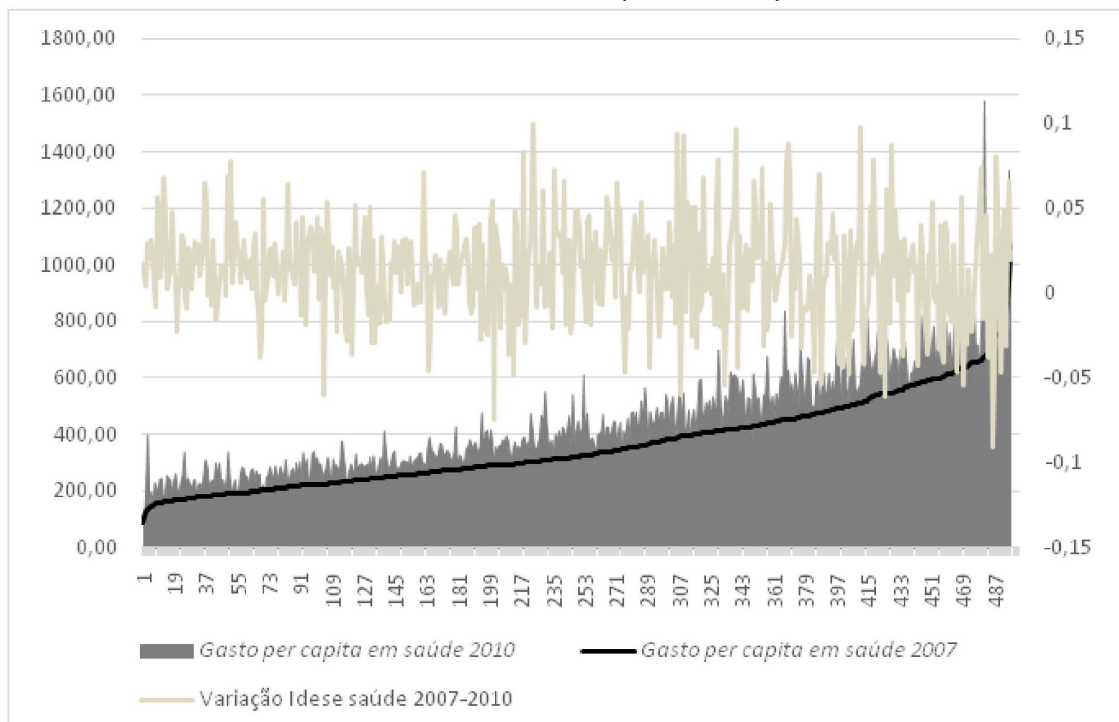

Fonte: Elaboração própria a partir de dados do Departamento de Informática do SUS (2014), da Fundação de Economia e Estatística do Rio Grande do Sul (2014) e do IBGE (2014).

Nota: Valores deflacionados pelo IPCA a preços de 2010.

Os resultados do Gráfico 1 mostram que, no período analisado, 125 municípios tiveram uma variação decrescente do Idese saúde, 46 não apresentaram variação e 325 mostraram variação positiva no período. O gasto médio em saúde per capita para o primeiro grupo foi de $\mathrm{R} \$ 87,69$, para o grupo que nãomostrouvariação foi de $R \$ 89,39$ e para o grupo que apresentou incremento no Idese saúde foi de $\mathrm{R} \$ 86,16$. Parece não haver relação direta entre o gasto per capita com saúde e as variações no Idese saúde. 
Gráfico 2 - Frequência de municípios do Rio Grande do Sul em relação à variação do Idese e do gasto médio per capita em saúde (2007-2010)

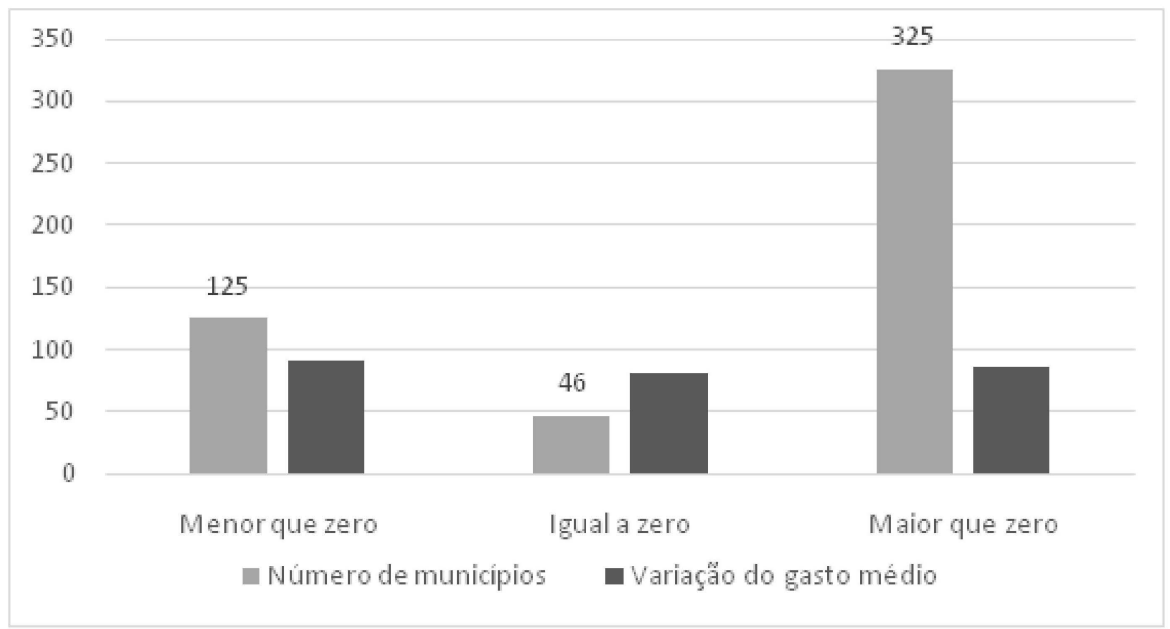

Fonte: Elaboração própria a partir de dados do Departamento de Informática do SUS (2014), da Fundação de Economia e Estatística do Rio Grande do Sul (2014) e do IBGE (2014).

Nota: Valores deflacionados pelo IPCA a preços de 2010.

De acordo com o Gráfico 2, para o ano de 2010 a média do Idese para os municípios do Rio Grande do Sul foi de 0,727, sendo esse considerado um nível médio de desenvolvimento. O nível alto seria acima de 0,8 e, o baixo, menor de 0,499 . Pode-se observar que os municípios enquadram-se no grupo de médio ou alto desenvolvimento, considerando-se apenas o Idese saúde. O menor valor variou de 0,678 , em 2007, para 0,666, em 2010, e o maior de 0,915,em 2007, para 0 , 924 em 2010.

Para se analisar o comportamento em relação às regiões e discutir o comportamento na desigualdade no estado, analisar-se-á os dados para os Coredes, criados em 1994, através da Lei no 10.283 , cujo objetivo é

[...] a promoção do desenvolvimento regional harmônico e sustentável; a integração dos recursos e das ações do governo na região; - a melhoria da qualidade de vida da população; - a distribuição equitativa da riqueza produzida; - o estímulo à permanência do homem em sua região; - a preservação e recuperação do meio ambiente (CONSELHO REGIONAL DE DESENVOLVIMENTO DA REGIÃO SUL, 2014). 

do Rio Grande do Sul (2007-2010)

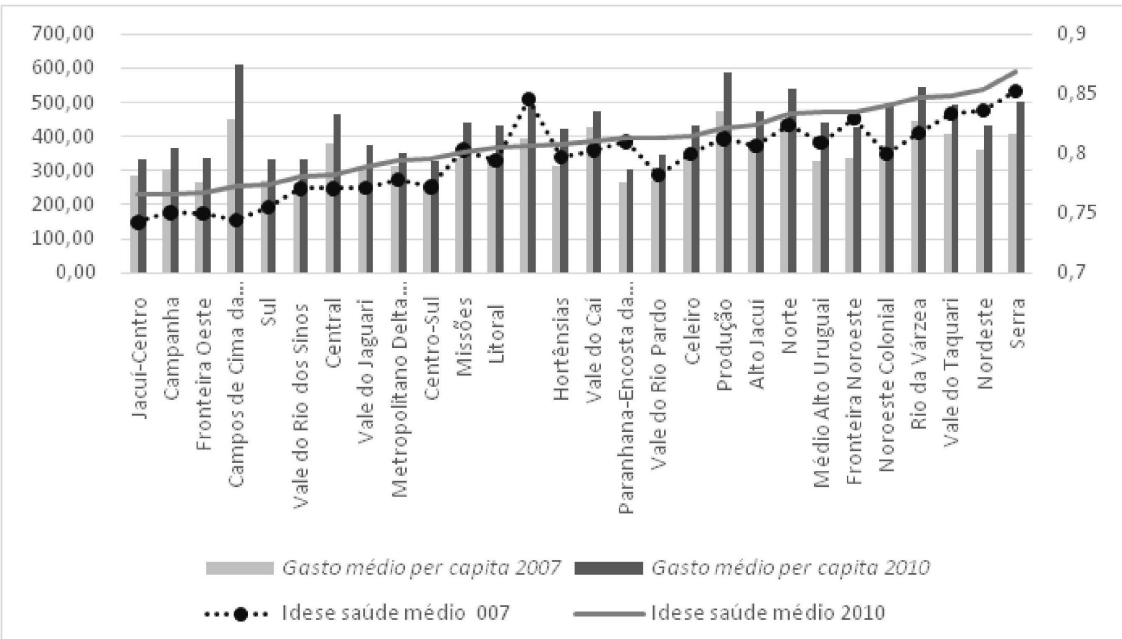

Fonte: Elaboração própria a partir de dados do Departamento de Informática do SUS (2014), da Fundação de Economia e Estatística do Rio Grande do Sul (2014) e do IBGE (2014). Nota: Valores deflacionados pelo IPCA a preços de 2010.

Os dados do Gráfico 3 foram ordenados pela coluna do Idese saúde de 2010. Como se pode observar, há uma tendência ao crescimento em todas as regiões do estado, com exceção da região do Alto da Serra de Botucaraí, onde o Idese saúde decresceu bastante, e da região das Missões, onde houve uma pequena diminuição, apesar de ter havido um aumento do gasto per capita em saúde. Em relação ao gasto per capita, houve aumento em todas as regiões em diferentes intensidades. A região que menos cresceu foi a da Paranhana-Encosta da Serra, com apenas $\mathrm{R} \$ 35,61$, e a que mais cresceu foi a dos Campos de Cima da Serra, com $R \$ 160,87$, sendo que a média de crescimento no estado foi de $R \$ 84,65$. As seguintes regiões cresceram abaixo da média do estado: Paranhana-Encosta da Serra, Metropolitano Delta do Jacuí, Vale do Caí, Jacuí Centro, Campanha, Sul, Vale do Rio dos Sinos, Vale do Jaguari, Fronteira Oeste, Nordeste, Centro-Sul e Vale do Rio Pardo. O crescimento diferenciado evidencia as desigualdades existentes entre as regiões, que também ocorre em relação a outros indicadores sociais. Estudos como os de Dachs (2002), Neri (2002) e Travassos (2006), que utilizam dados da PNAD para estudar a desigualdade de acesso aos serviços de saúde (públicos e privados), estabelecendo uma relação com variáveis como renda e escolaridade, mostram que o Rio Grande do Sul destaca-se pela magnitude das desigualdades sociais no acesso aos serviços de saúde. No Gráfico 4 podemos observar o comportamento do Idese para essas regióes. 

Coredes do Rio Grande do Sul (2007-2010)

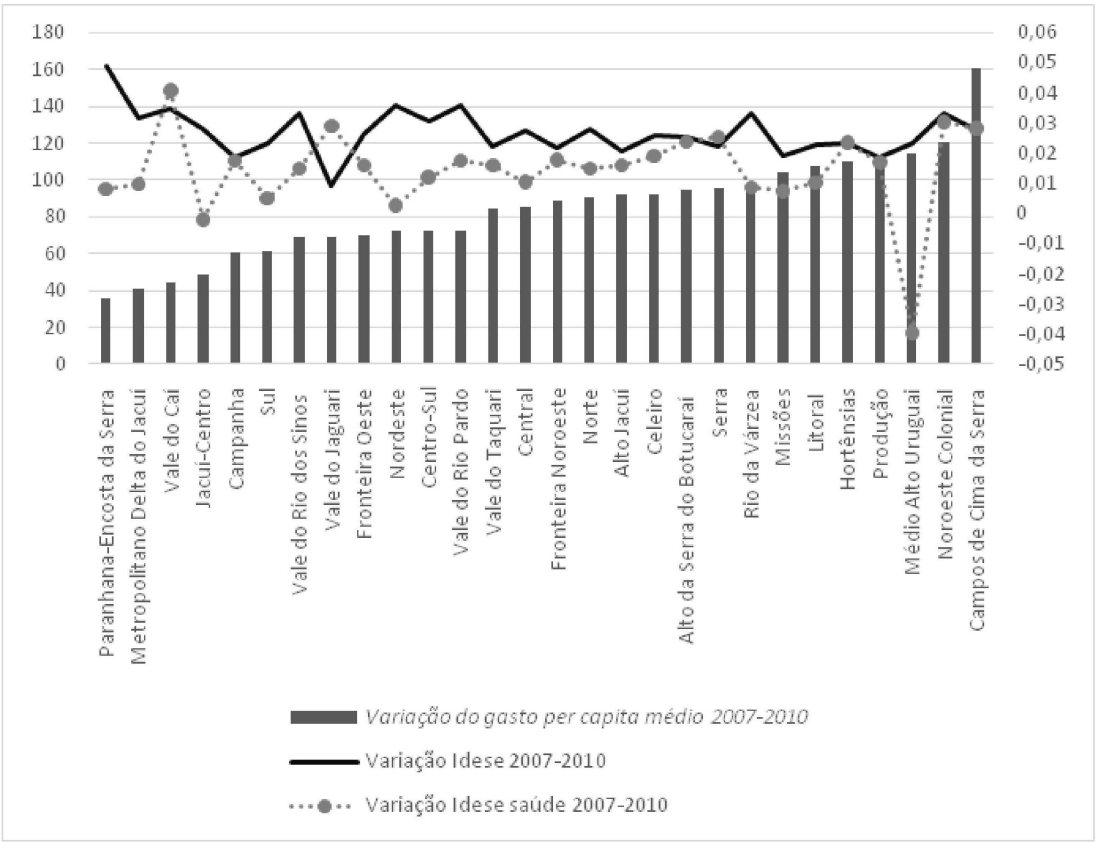

Fonte: Elaboração própria a partir de dados do Departamento de Informática do SUS (2014), da Fundação de Economia e Estatística do Rio Grande do Sul (2014) e do IBGE (2014). Nota: Valores deflacionados pelo IPCA a preços de 2010.

No Gráfico 4, os Coredes foram ordenados de acordo com a variação do gasto médio per capita em saúde no período e analisados em relação à variação do Idese e do bloco saúde do Idese. Em relação à variação do gasto médio per capita em saúde, os dados mostram que em todos os Coredes houve aumento do gasto médio, que variou entre $\mathrm{R} \$$ 35,61 e R $\$ 160,87$ nos Coredes Paranhama e Campo do Alto da Serra, respectivamente.

Em relação ao Idese, também houve crescimento no período de 0,009, no Vale do Jacuí, e de 0,049 no Paranhama. Para o Idese saúde, no entanto, o comportamento não foi o mesmo e, em dois deles, Alto da Serra do Botucaraí e Missões, houve diminuição no período. Para os outros dois não havia informação para o ano de 2007, e o restante cresceu entre 0,003 e 0,041 no período. $O$ aumento do gasto médio em saúde não parece impactar o Idese e tampouco o Idese saúde, sendo que o coeficiente de correlação entre a variação do gasto médio em saúde e o Idese foi de 0,03 , portanto a correlação pode ser considerada bem fraca. Entre o gasto médio em saúde per capita e o Idese saúde foi de 0,12 , uma correlação que também pode ser considerada bem fraca. $\mathrm{O}$ coeficiente de correlação foi calculado com base nas variações após a exclusão dos dois Coredes, quando não havia mais informações para o ano de 2007. 


\section{Considerações Finais}

Este estudo parece comprovar a importância da construção de políticas públicas convergentes para a criação de condiçōes econômicas e sociais que promovam o bem-estar. A saúde é certamente um fator intrínseco ao desenvolvimento social e econômico e pode ser vista como um determinante, uma medida e um resultado do progresso, mas essas condiçōes são muitas vezes produzidas fora dos sistemas de saúde, sendo resultado do conjunto de políticas públicas, como educação, agricultura, transporte, meio ambiente e outras políticas econômicas e sociais UNITED NATIONS RESEARCH INSTITUTE FOR SOCIAL DEVELOPMENT, 2013). Em outras palavras, as políticas de saúde isoladamente parecem não ser capazes de afetar as condições de bem-estar e saúde.Os gastos realizados com saúde não resultaram em melhorias diretas das condições de saúde ou bem-estar medidas pelo Idese, ou mesmo pelo Idese saúde. Tampouco foram capazes de reduzir as desigualdades entre as regióes. Existe no período uma tendência ao crescimento do Idese saúde que não parece estar relacionado com o gasto realizado.

É necessário ponderar algumas questôes que poderiam explicar essa situação. Em primeiro lugar, a literatura aponta que séries de dados de períodos mais longos seriam mais indicadas para avaliar esse tipo de política pública. Infelizmente ainda não existem informaçōes disponíveis para períodos maiores. Deve-se considerar se revisitar os dados em alguns anos para confirmar ou não essas conclusões. Em segundo lugar, é necessário considerar a organização do sistema de saúde como um modelo hierarquizado, ou seja, os municípios maiores são referência regional e podem apresentar suas informaçōes de gasto per capita distorcidas, pois, de fato, atendem uma populaçăo maior. Por fim, deve-se analisar a forma de financiamento do sistema, que é mais focada na oferta de serviços, entre outras questões que já são apontadas desde a década de 1950. O Instituto de Pesquisas para o Desenvolvimento Social das Naçôes Unidas (196819 apud DUARTE DE ARAÚJO, 1975, p. 519) e o Relatório da Comissăo Nacional de Bem-Estar Social de 1954 (1954 ${ }^{20}$ apud DUARTE DE ARAÚJO, 1975, p. 523) já chamavam atenção para a capacidade da assistência médica, como fator isolado da ação de saúde, resultar na melhoria da qualidade de vida da população, criticando a tendência de construir uma "superestrutura médica" e apontando o risco de dispersão de recursos, de múltiplas ações e de falta de organização na formação de profissionais. Ou, ainda, chamavam atenção para a subordinaçăo às teses neoliberais que propõe o Estado mínimo (FLEURY, 2009; GALLO, 2005; MACIEIRA, 2007; MENICCUCCI,

19 UNITED NATIONS. Research Institute for Social Development. Geneva: UN, 1968. (Research Notes, n. 1).

20 CASTRO, A. et al. Saúde pública e desenvolvimento econômico. Relatório da Subcomissáo de Saúde da Comissáo Nacional de Bem-Estar Social. Revista Hig. Saúde Publica, Rio de Janeiro, v. 13, p. 53-64, 1954. 
2007; SANTOS, 2008) com todas as suas consequências, como o investimento insuficiente na proteção social que resultou em um desastre social nas décadas seguintes (JOMO, 2003), em decorrência da incapacidade dos países de se contrapor às rápidas mudanças estruturais e aos ciclos econômicos.

No Brasil, as políticas de descentralização e regionalização do SUS realizaram grandes investimentos em infraestrutura assistencial (obras e equipamentos) desigual entre as regiōes (SILVA JÚNIOR, 1998; SCHRAIBER, 1995), favorecendo os serviços privados através de financiamento público e organizando um sistema de saúde mais focado na oferta de serviços.

\section{Referências}

BLAS, E. et al. Adressing social determinants of health inequities: what can state and civil society do? The Lancet, v. 372, n. 9650, p. 1684-1689, 2008.

BORRELL, C.; ARTAZCOZ, L. Las Políticas para disminuir las desigualdades en salud. Gaceta Sanitaria, v. 22, n. 5, p. 465-473, Sept//Oct. 2008.

CASTRO, J. D. A utilização do sistema de custeio por absorção para avaliar custos da atenção básica de saúde: reformulações e aprimoramentos metodológicos. 2000. $213 \mathrm{f}$. Tese (Doutorado em Saúde Coletiva) - Programa de Pós-Graduação em Saúde Coletiva, Faculdade de Ciências Médicas, Universidade Estadual de Campinas, Campinas, SP, 2000.

CONSELHO REGIONAL DE DESENVOLVIMENTO DA REGIÃO SUL. Secretaria de Planejamento Gestão e Participaçāo Cidadā. O que são e como funcionam os Coredes RS. 2014. Disponivel em: <http://www.coredesul.org.br/Pagina/9/O-QUE-SAO-E-COMOFUNCIONAM-OS-COREDES-\%C2\%96RS>. Acesso em: 5 jun. 2014.

DACHS, N. Determinantes das desigualdades na auto-avaliaçăo de saúde no Brasil: análise dos dados da PNAD/1998. Ciência \& Saúde Coletiva, Rio de Janeiro, v. 7, n. 4, p. 641-657, 2002.

DEPARTAMENTO DE INFORMÁTICA DO SUS. SIOPS - Sistema de Informações sobre Orçamento Público em Saúde. Disponivel em: <http://datasus.saude.gov.br/sistemas-eaplicativos/financeiros/siops >. Acesso em: $30 \mathrm{dez} .2014$.

DRACHLER, M. L. et al. Proposta de metodologia para selecionar indicadores de desigualdade em saúde visando definir prioridades de políticas públicas no Brasil. Ciência \& Saúde Coletiva, Rio de Janeiro, v. 8, n. 2, p. 462-479, 2003.

DUARTE DE ARAÚJO, J. D. Saúde e desenvolvimento econômico: atualização de um tema. Revista de Saúde Pública, São Paulo, v. 9, p. 515-528, 1975.

FLEURBAEY, M.; SCHOKKARERT,E. Unfair inequalities in health and health care. Joumal of Health Economics, v. 28, n. 1, p. 73-90, Jan. 2009.

FLEURY, S. Reforma sanitária Brasileira: dilemas entre o instituinte e o instituído. Ciência $\mathbb{E}$ Saúde Coletiva, Rio de Janeiro, v. 14, n. 3, p. 743-753, maio/jun. 2009. 
FUNDAÇÃO DE ECONOMIA E ESTATÍSTICA DO RIO GRANDE DO SUL. Disponível em: <http://www.fee.rs.gov.br>. Acesso em: 30 dez. 2014.

GADELHA, C. A. G. Desenvolvimento, complexo industrial da saúde e política industrial. Revista de Saúde Pública, v. 40, n. especial, p. 11-23, 2006.

GADELHA, C. A. G. et al. Saúde e territorialização na perspectiva do desenvolvimento. Ciência e Saúde Coletiva, Rio de Janeiro, v. 16, n. 6, p. 3003-3016, jun. 2011.

GALLO, E. et al. Saúde,desenvolvimento e globalização. Saúde em Debate, Rio de Janeiro, v. 29, n. 71, p. 315-326, set/dez. 2005.

GUIMARĀES, J. R. S.; JANUZZI, P. M. Indicadores sintéticos no processo de formulaçăo e avaliação de políticas públicas: limites e legitimidades. In: ENCONTRO NACIONAL DE ESTUDOS POPULACIONAIS, 14., 2004, Caxambu. Anais... Caxambu: ABEP, 2004.

IBGE. Índice Nacional de Preços ao Consumidor Amplo - IPCA e Índice Nacional de Preços ao Consumidor - INPC. Disponfvel em: < http://www.ibge.gov.br/home/estatistica/indicadores/ precos/inpc_ipca>. Acesso em 30 dez. 2014.

JOMO, K. S. Globalization, liberalization and equitable development: lessons from East Asia. Geneva: UNRISD, June 2003. (Paper n. 3).

LUIZ, O. C. et al. Diferenciais intermunicipais de condiçóes de vida e saúde: construçáo de um indicador composto. Revista Saúde Pública, v. 34, n. 1, p. 115-122, fev. 2009.

MACIEIRA, D. Actores y reformas em salud en América Latina. Buenos Aires: Banco Interamericano de Desarrollo, 2007. (Nota Técnica de Salud, 1).

MARMOT, M. Achieving health equity: from root causes to fair outcomes. The Lancet, v. 370, n. 9593, p. 1153-1163, Sept. 2007.

MÉDICI, A. C. et al. Sistemas de custos como instrumento de eficiência e qualidade dos serviços de saúde. São Paulo: Fundap/IESP, 1995.

MENICCUCCI,T. M. G. Implementação da reforma sanitária: formação de uma política. Saúde e Sociedade, Säo Paulo, v. 15, n. 2, p. 72-87, maio/ago. 2006.

MIRVIS, D. M.; CHANG,C. The relationship between health and development: health as an economic engine. Journal of Health and Human Services Administration, v. 31, n. 1, p. 30-57, 2008.

MOLLO, M. L. R.; FONSECA, P. C. D. Desenvolvimento e novo desenvolvimentismo: raizes teóricas e precisōes conceituais. Revista de Economia Política, São Paulo, v. 33, n. 2, p. 222239, abr.jun. 2013.

MOREL, C. A pesquisa em saúde e os objetivos do milênio: desafios e oportunidades globais, soluçöes e políticas nacionais. Ciência \& Saúde Coletiva, v. 9, n. 2, p. 261-270, 2004.

NERI, M.; SOARES, W. Desigualdade social e saúde no Brasil. Cadernos de Saúde Pública, Rio de Janeiro, v. 18, p. 77-88, 2002. (Suplemento). 
PELEGRINI, M. L. M.; CASTRO, J. D. Eqüidade na alocação de recursos para a saúde: a experiência do estado do Rio Grande do Sul, Brasil. Ciência \& Saúde Coletiva, Rio de Janeiro, v. 10, n. 2, p. 275-286, abr/jun. 2005.

PIOLA, S. F.; VIANNA, S. M. (Org.). Economia da saúde: conceitos e contribuição para a gestão em saúde. Brasilia: Ipea, 1995.

SANTOS, N. R. Política pública de saúde no Brasil: encruzilhada, buscas e escolhas de rumos. Ciencia e Saúde Coletiva, Rio de Janeiro, v. 13, p. 2009-2018, 2008. (Suplemento 2).

SCHRAIBER, L. B. Políticas públicas e planejamento nas práticas de saúde. Saúde em Debate, v. 47, n. 1, p. 28-35, jan/abr. 1995.

SEN, A. ¿Por qué la equidad en salud? Revista Panamericana de Salud Pública, v. 11, n. 5/6, p. 302-309, 2002.

SILVA JUNIOR, A. G. Modelos tecnoassistenciais em saúde: o debate no campo da saúde coletiva. Säo Paulo: Hucitec, 1998.

TRAVASSOS, P.; OLIVEIRA, E.; VIACAVA,F. Desigualdades geográficas e sociais no acesso aos serviços de saúde no Brasil: 1998 e 2003. Ciência E Saúde Coletiva, Rio de Janeiro, v. 11 , n.4, p. 975-986, 2006.

UNITED NATIONS RESEARCH INSTITUTE FOR SOCIAL DEVELOPMENT. Health Systems as Social Institutions: progress towards health in all policies. May 2013. Disponivel em: <http://goo.gl/yMLgcX>. Acesso em: 12 ago. 2014.

VIANA, A.L.D.; ELIAS, P. E. M. Saúde e desenvolvimento. Ciência e Saúde Coletiva, v. 12, p. 1765-1777, 2007. (Suplemento).

WORLD HEALTH ORGANIZATION. Final report commission on the social determinants of health: closing the gap in a generation. Geneva: WHO, 2008.

Recebido em: 21/06/2011. Aceito em: 29/12/2014. 